\title{
ESTUDO DO EFEITO DO APOIO VISUAL DO TRAÇADO ESPECTROGRÁFICO NA CONFIABILIDADE DA ANÁLISE PERCEPTIVO-AUDITIVA
}

\author{
Studying the effect of spectrogram visual support of \\ in the auditory-perceptive voice evaluation reliability
}

\author{
Ana Cristina Côrtes Gama (1), Luiza Lara Marques Santos ${ }^{(2)}$, Natália Aparecida Sanches ${ }^{(3)}$, \\ Marcela Guimarães Côrtes ${ }^{(4)}$, lara Barreto Bassi ${ }^{(5)}$
}

\begin{abstract}
RESUMO
Objetivo: avaliar a concordância intra e inter-sujeitos na avaliação perceptivo-auditiva realizada de forma isolada e simultaneamente à apresentação do traçado espectrográfico correspondente, a fim de verificar se a apresentação simultânea dos estímulos vocais e espectrográficos aumenta a concordância da avaliação perceptivo-auditiva da voz. Método: trata-se de um estudo longitudinal, em que seis fonoaudiólogas avaliaram, em dois momentos distintos, 105 vozes disfônicas e não disfônicas, de forma perceptivo-auditiva: primeiramente sem e posteriormente com a apresentação dos espectrogramas correspondentes. Vinte por cento das vozes foram repetidas aleatoriamente nos dois momentos, a fim de se analisar as concordâncias intra-avaliadoras. Utilizou-se a escala GRBASI para realização da avaliação vocal. Para análise da concordância inter-avaliadores, foi utilizado o índice estatístico Kappa Fleiss e, para cálculo da concordância intra-avaliador, foi utilizado o coeficiente de correlação de Spearman. Resultados: não houve diferença estatisticamente significante entre as avaliações perceptivo-auditivas inter-sujeitos com e sem a leitura espectrográfica, porém houve aumento da concordância entre os avaliadores para as variáveis $G, R, B$ e S. Não houve diferença estatisticamente significante entre as avaliações perceptivo-auditivas intra-sujeitos sem e com o apoio visual do espectrograma, entretanto, houve aumento da concordância intra-avaliadores após a apresentação do estímulo visual, para as variáveis G, B e I. Conclusão: o apoio visual do espectrograma não aumenta significativamente a confiabilidade da avaliação perceptivo-auditiva da voz, mas a auxilia, uma vez que promove um aumento da concordância inter e intra-avaliadores.
\end{abstract}

DESCRITORES: Voz; Distúrbios da Voz; Fonoaudiologia; Acústica da Fala

(1) Fonoaudióloga; Professora Adjunto do Departamento de Fonoaudiologia da Universidade Federal de Minas Gerais/ UFMG; Belo Horizonte - MG.

(2) Fonoaudióloga Clínica, graduada em Fonoaudiologia pela Universidade Federal de Minas Gerais/UFMG; Belo Horizonte - MG.

(3) Fonoaudióloga Clínica, graduada em Fonoaudiologia pela Universidade Federal de Minas Gerais/UFMG; Belo Horizonte - MG.

(4) Fonoaudióloga; Mestranda em Saúde da Criança e do Adolescente do Departamento de Pediatria da Universidade Federal de Minas Gerais/UFMG; Belo Horizonte - MG.

(5) Fonoaudióloga; Mestranda em Saúde e Trabalho pelo Programa de Pós-graduação em Saúde Pública do Departamento de Medicina Preventiva e Social da Faculdade de Medicina da Universidade Federal de Minas Gerais/UFMG; Belo Horizonte - MG.

Conflito de interesses: inexistente

\section{INTRODUÇÃO}

A voz é produzida no trato vocal e é resultado da integração entre a fonação e a ressonância. É, portanto, um atributo peculiar ao ser humano e, além dos aspectos fisiológicos, funciona como um veículo de expressão da personalidade, das emoções e até mesmo do estado psico-emocional do indivíduo. Assim sendo, a voz é uma manifestação com base psicológica mas de sofisticado processamento muscular ${ }^{1}$.

Não existem definições exatas ou unanimamente aceitas sobre as características da voz normal, de modo que não há padrões ou limites definidos a este respeito². Quando a harmonia muscular é mantida, 
os ouvintes atribuem boa qualidade ao som e o falante a produz sem dificuldade ou desconforto. Por outro lado, emissões ruidosas e produzidas com certo grau de desconforto podem configurar uma disfonia. Sendo assim, a disfonia é um sintoma decorrente de desajustes na fonação que podem estar relacionados a diversas etiologias ${ }^{1}$.

A avaliação fonoaudiológica mostra-se como instrumento eficaz para a análise dos distúrbios vocais. A partir da avaliação da voz, pode-se descrever o perfil vocal do indivíduo e identificar os fatores desencadeadores ou mantenedores do quadro disfônico ${ }^{1}$. Existem várias formas de avaliação vocal, as mais preconizadas na clínica fonoaudiológica são a análise perceptivo-auditiva e a análise acústica da voz.

A avaliação perceptivo-auditiva é a avaliação tradicional na rotina clínica fonoaudiológica. Embora críticas sejam feitas à subjetividade e à imprecisão terminológica envolvida neste procedimento ${ }^{3,4}$, ela é considerada padrão ouro para avaliação da qualidade vocal. A avaliação perceptivo-auditiva permite a inferência de dados anatomofisiológicos importantes e pode fornecer informações sobre os aspectos psicossociais da voz.

A análise perceptivo-auditiva é um teste subjetivo que se baseia especialmente na impressão do avaliador sobre a emissão vocal do indivíduo, sofrendo influência do nível de experiência do mesmo neste tipo de avaliação, do material de voz analisado, do tipo de apresentação, do grau de desvio da qualidade vocal, além do tipo de escala utilizada nesta tarefa ${ }^{5}$.

A escala GRBAS é uma escala perceptivo auditiva criada pelo Commitee for Phonatory Function Tests da Japan Society of Logopedics and Phoniatrics e divulgada por Hirano $(1981)^{6} \mathrm{e}$, tornouse bastante utilizada para mensurar vozes disfônicas devido à sua praticidade e grau de confiabilidade $e^{7-9}$. Por meio desta, é possível determinar o grau global da disfonia $(G)$ pela identificação de quatro fatores independentes: R (rugosidade), B (soprosidade), A (astenia) e S (tensão). O parâmetro "I" para instabilidade foi adicionado posteriormente Dejonckere et al $(1996)^{7}$ e relaciona a qualidade da voz ao seu grau de flutuação, assim formando a escala GRBASI.

A avaliação acústica quantifica o sinal sonoro, o que torna a análise vocal mais objetiva e pode basear-se em formas distintas de avaliação: na extração de medidas acústicas, na espectrografia vocal, dentre outras ${ }^{10}$.

A análise espectrográfica mede a acústica da onda sonora vocal e a registra em um gráfico tridimensional denominado espectrograma ${ }^{1}$, desta forma, o espectrograma promove uma visualização da fala, assim algumas vezes referido como "visão da fala" (visible speech) ${ }^{10}$.
O espectrograma pode ser definido como um gráfico tridimensional que registra características de duração, intensidade e freqüência da voz, de modo que as intensidades relativas dos componentes da onda sonora são indicadas pelo escurecimento ou coloração de faixas de freqüência (eixo vertical) em função do tempo (eixo horizontal). A partir desta avaliação, o especialista pode inferir aspectos da função glótica durante a emissão vocal. Apesar de ser uma avaliação objetiva, o espectrograma necessita da interpretação dos avaliadores para as informações apresentadas por ela ${ }^{11,12}$. A avaliação espectrográfica depende, portanto, de uma análise perceptivo-visual, sofrendo influências da experiência do avaliador.

Vale ressaltar a existência de estudos na literatura que contemplam em seus resultados bom nível de confiabilidade para a avaliação vocal, quando esta é realizada por meio da GRBASI ${ }^{7,8}$. Assim como outro, que menciona a importância do treinamento auditivo dos avaliadores para obtenção de resultados mais confiáveis ${ }^{9}$. Por fim, os estudos que enfocam a utilização da avaliação perceptivaauditiva somada ao apoio visual espectrográfico, realçam a relevância da associação destas avaliações, já que as mesmas trazem informações complementares sobre a qualidade vocal ${ }^{10,12,13}$.

Assim, a confiabilidade de tais julgamentos continuam sendo um assunto central no estudo da avaliação da qualidade da voz e dos distúrbios vocais ${ }^{4}$ e, estudos que procurem analisar estratégias para melhorar tais concordâncias são importantes no estudo de vozes disfônicas e na clínica fonoaudiológica.

O objetivo do presente estudo é avaliar a concordância intra e inter-sujeitos na avaliação perceptivoauditiva realizada de forma isolada e simultânea ao traçado espectrográfico correspondente, a fim de verificar se a análise dos estímulos vocais associada ao apoio visual do traçado espectrográfico aumenta a concordância e conseqüentemente a confiabilidade da avaliação perceptivo-auditiva da voz.

\section{MÉTODO}

Trata-se de um estudo longitudinal prospectivo no qual foram avaliadas de forma perceptivo-auditiva vozes disfônicas e não disfônicas, sem e com o apoio visual do traçado espectrográfico. Seis fonoaudiólogas com experiência em voz foram convidadas a participar da pesquisa após a assinatura do Termo de Consentimento Livre e Esclarecido.

Foram selecionadas 105 vozes de indivíduos maiores de 18 anos, de ambos os sexos, disfônicos e não disfônicos, pertencentes a um banco 
de vozes de um consultório particular. Todos os pacientes foram devidamente orientados sobre sua participação na pesquisa e permitiram a utilização de suas vozes após assinarem o Termo de Consentimento Livre e Esclarecido.

O material de voz utilizado na pesquisa foi a emissão da vogal /a/ sustentada e uma amostra de fala automática (contagem de 1 a 20 ou meses do ano). $\mathrm{Na}$ ocasião da gravação, solicitou-se ao paciente que inspirasse e emitisse a vogal /a/ prolongada em frequência habitual de fala, seguido da fala automática.

Para o registro do material, utilizou-se um microfone profissional, do tipo condensador, estéreo, omni-direcional, sensitividade de $-20 \mathrm{~dB}$ da marca EQUITEK E-100, ligado a uma fonte de eletricidade (Phanton Power) de uma mesa de som marca MACKIE $^{\text {TM }} 1202$ VLZ - 12 canais. Os indivíduos estavam de pé, com o microfone situado a $10 \mathrm{~cm}$ da boca e com ângulo de captação direcional de $90^{\circ}$. O microfone estava deslocado do corpo da unidade de gravação para evitar captação de ruído do maquinário.

As gravações foram feitas em ambiente silente, com ruído ambiental inferior a 50dB NPS (nível de pressão sonora), aferido por meio de um medidor de nível de pressão sonora digital marca RÁDIO SHACK $^{\text {TM }}$ (cat. N. ${ }^{\circ}$ 33-2055). Utilizou-se um PC IBM Aptiva E30P'M, processador AMD - K6 - 2/500 $\mathrm{MHz}$, memória de 128 mega bytes RAM, espaço de disco de 8,4 Giga bytes, placa de som Crystal SoudFusion ${ }^{\mathrm{TM}}$.

A captação das vozes foi realizada com o programa "Gravador de som" disponível no Windows XP. Os arquivos foram salvos em formato Wave $(44100 \mathrm{~Hz}, 16$ Bits, mono) e em seguida, gravados em CD marca Sony.

As emissões das vogais sustentadas foram transferidas para o programa Gram 5.0, o qual gera a imagem espectrográfica do som. Os espectrogramas foram gerados em mono, sample rate de $11 \mathrm{k}$, resolução de 16 bit, escala de $60 \mathrm{~dB}$, display scroll e paleta $C B$. A análise das frequências foi realizada em escala linear, banda estreita, FFT 1024 e resolução $5.4 \mathrm{~Hz}$.

A amostra, composta pelo número de avaliadores e de vozes analisadas, foi definida a partir do índice de Kappa proposto por Fleiss, com um poder estatístico de $80 \%$ e nível de significância de 5\%. A fim de se determinar a concordância intra-avaliadores, $20 \%$ das vozes e dos traçados espectrográficos foram repetidos, aleatoriamente, totalizando 126 vozes e espectrogramas.

As seis fonoaudiólogas convidadas a avaliar as vozes possuíam experiência na área de voz há mais de um ano ( 01 a 15 anos) e idade entre 24 e
44 anos, com média de 32 anos. Para realização das avaliações, foi preenchido, individualmente e por escrito, o protocolo de avaliação contendo a escala GRBASI, proposta por Hirano $(1981)^{6} \mathrm{e}$ acrescida do parâmetro (I) por Dejonckere (1996) ${ }^{7}$. Esta escala permite analisar os seguintes aspectos da qualidade vocal: rugosidade da voz $(R)$, soprosidade $(B)$, astenia $(A)$, tensão $(S)$ e instabilidade $(I)$, que em seu conjunto determinam o grau geral da disfonia (G). Cada um desses aspectos pode ser classificado em uma escala de severidade de 0 a 3 , sendo 0 sem alteração; 1 levemente alterado; 2 moderadamente alterado e 3 alteração severa. Antes das análises, as avaliadoras foram treinadas e esclarecidas sobre a forma de preenchimento da escala para garantir uma maior uniformidade entre suas avaliações.

As avaliações ocorreram em dois momentos, sendo que no primeiro encontro as 126 amostras constituídas da vogal /a/ sustentada e fala automática foram apresentadas no programa Windows Media Player, por meio de fone de ouvido da marca Hypson, plugado a um notebook HP Pavilion $d v 6000$, e classificadas pelas avaliadoras por meio da escala GRBASI. No segundo encontro, as 126 amostras de voz e fala foram novamente avaliadas, simultaneamente à apresentação dos traçados espectrográficos correspondentes a cada emissão.

A fim de reduzir a possibilidade de memorização, os encontros foram realizados com um intervalo mínimo de dois meses, sendo o primeiro encontro entre dezembro de 2008 e janeiro de 2009 e o segundo entre março e abril do mesmo ano. Os encontros foram realizados em consultórios do Ambulatório de Fonoaudiologia do Hospital das Clínicas da UFMG. As avaliadoras não obtiveram quaisquer informações a respeito dos pacientes cujas vozes foram avaliadas, tais como nome, idade, sexo e alteração laríngea.

Este projeto foi aprovado pelo Comitê de Ética em Pesquisa da UFMG - COEP- UFMG, sob parecer de número ETIC 405/08.

Por meio dos protocolos preenchidos em cada encontro, foi testada a concordância intra e interavaliadores para a análise perceptivo-auditiva com e sem o traçado espectrográfico, a partir de análise qualitativa e quantitativa dos resultados.

Todas as análises foram realizadas no Software STATA, versão 9.0, (Stata Corporation, College Station, Estados Unidos). Foi adotado o nível de significância de 5\% em todos os testes.

Para análise da concordância inter-avaliadores foi utilizado o índice estatístico Kappa Fleiss e considerada a classificação de concordância proposta por Landis \& Koch: (a): quase perfeita: kappa entre 0,80 e 1,00; (b): boa: kappa entre 0,60 e 0,80; (c): 
moderada: kappa entre 0,40 e 0.60; (d): regular: kappa entre 0,20 e 0,40; (e): discreta: kappa entre zero e 0,20; (f): pobre: kappa entre -1 e zero.

Para cálculo da concordância intra-avaliador foi utilizado o coeficiente de correlação de Spearman, que varia de -1 a 1.

\section{RESULTADOS}

Os resultados descritos abaixo indicam que não houve diferença estatisticamente significante entre as avaliações perceptivo-auditivas intersujeitos com e sem a leitura espectrográfica. Desta forma, pode-se dizer que o apoio visual do traçado espectrográfico não aumenta a confiabilidade da avaliação perceptivo-auditiva da qualidade vocal, entretanto, aumenta a concordância entre avaliadores para as variáveis $G, R, B$ e $S$ (Tabela 1 e Figura 1). $O$ apoio visual do espectrograma, portanto, auxilia a avaliação perceptivo-auditiva da qualidade vocal, sem, entretanto, aumentar sua confiabilidade.

Tabela 1 - Resultados da análise da concordância inter-sujeitos na avaliação perceptivo-auditiva com e sem apoio visual

\begin{tabular}{cccccc}
\hline \multirow{2}{*}{ VARIÁVEL } & \multicolumn{2}{c}{ SEM APOIO VISUAL } & \multicolumn{2}{c}{ COM APOIO VISUAL } & \multirow{2}{*}{ P-valor } \\
\cline { 2 - 5 } & Kappa Fleiss & IC95\% & Kappa Fleiss & IC95\% & \\
\hline G & $0.44(\mathrm{c})$ & $(0.38-0.50)$ & $0.50(\mathrm{c})$ & $(0.44-0.56)$ & 0.138 \\
R & $0.31(\mathrm{~d})$ & $(0.26-0.36)$ & $0.32(\mathrm{~d})$ & $(0.26-0.37)$ & 0.791 \\
B & $0.18(\mathrm{e})$ & $(0.13-0.23)$ & $0.25(\mathrm{~d})$ & $(0.19-0.30)$ & 0.087 \\
A & $0.14(\mathrm{e})$ & $(0.07-0.22)$ & $0.10(\mathrm{e})$ & $(0.05-0.15)$ & 0.320 \\
S & $0.23(\mathrm{~d})$ & $(0.16-0.29)$ & $0.29(\mathrm{~d})$ & $(0.21-0.37)$ & 0.207 \\
I & $0.25(\mathrm{~d})$ & $(0.18-0.31)$ & $0.23(\mathrm{~d})$ & $(0.16-0.28)$ & 0.640 \\
\hline
\end{tabular}

G: Grau geral da disfonia; I: Instabilidade; R: Rugosidade; B: Soprosidade; A:Astenia; S: Tensão. p-valor $<0,05$ (significância estatística).

IC95\%: Intervalo de confiança de 95\%.

Estatística: Kappa fleiss

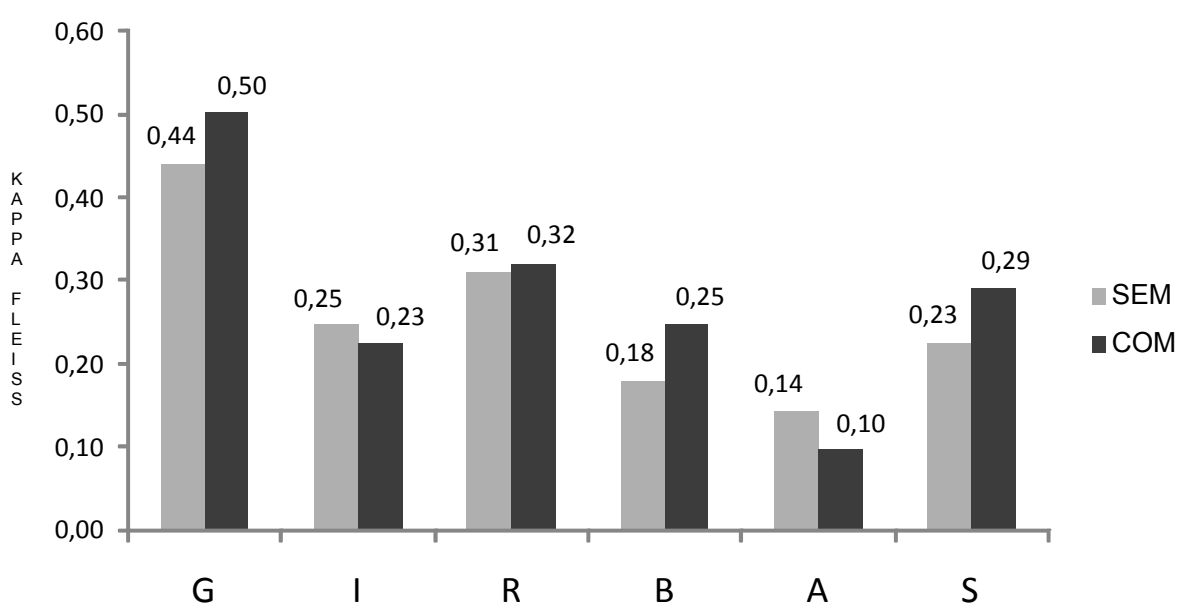

G: Grau geral da disfonia; I: Instabilidade; R: Rugosidade; B: Soprosidade; A:Astenia; S: Tensão.

Estatística: Kappa fleiss

Figura 1 - Representação do índice estatístico Kappa da concordância inter-sujeitos da análise perceptivo-auditivas, com e sem apoio visual. 
A Tabela 2 e a Figura 2 correspondente demonstram que não houve diferença estatisticamente significante entre as avaliações perceptivo-auditivas intra-sujeitos sem e com o apoio visual do espectrograma, entretanto, houve aumento da concordância intra-avaliadores após a apresentação do estímulo visual para as variáveis $\mathrm{G}$, B e I. Da mesma forma como observado para os valores inter-avaliadores, o apoio visual do espectrograma auxilia a avaliação perceptivo-auditiva da qualidade vocal, sem, entretanto, aumentar sua confiabilidade.

Tabela 2 - Resultados da análise da concordância intra-avaliador na avaliação perceptivo-auditiva sem e com apoio visual

\begin{tabular}{lcccccc}
\hline VARIÁVEL & G & R & B & A & S & I \\
\hline Sem apoio visual & 0.83 & 0.81 & 0.61 & 0.83 & 0.76 & 0.80 \\
Com apoio visual & 0.86 & 0.71 & 0.72 & 0.60 & 0.76 & 0.85 \\
P-valor & 0.60 & 0.12 & 0.35 & 0.17 & 0.92 & 0.25 \\
\hline
\end{tabular}

G: Grau geral da disfonia; I: Instabilidade; R: Rugosidade; B: Soprosidade; A:Astenia; S: Tensão.

Estatística: Spearman

Wilcoxon: $p$-valor utilizado para cálculo da significância estatística $(p<0,05)$.

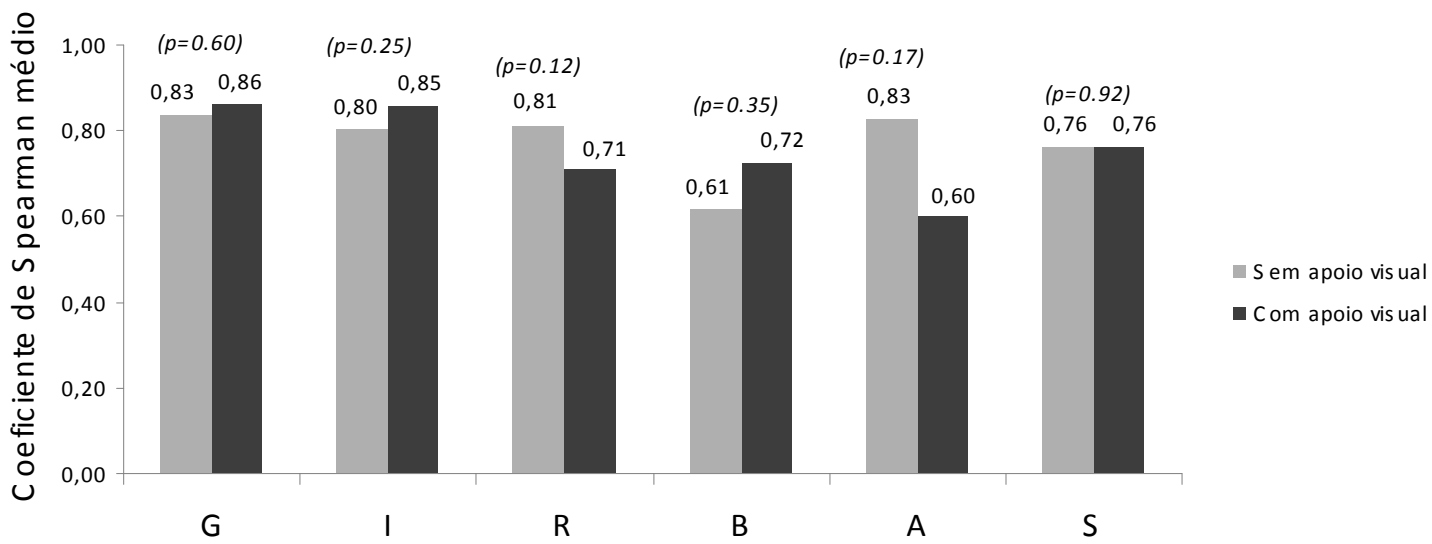

G: Grau geral da disfonia; I: Instabilidade; R: Rugosidade; B: Soprosidade; A:Astenia; S: Tensão. Estatística: Spearman

Wilcoxon: $p$-valor utilizado para cálculo da significância estatística $(p<0,05)$.

Figura 2 - Representação dos valores estatísticos do Coeficiente de Sperman da concordância intrasujeitos da análise perceptivo-auditivas, com e sem apoio visual.

\section{DISCUSSÃO}

A análise perceptivo-auditiva da voz é soberana na prática clínica, especialmente por traduzir de forma mais fidedigna os aspectos relacionados à qualidade vocal. Entretanto, apresenta um grau de subjetividade que pode vir a comprometer a confiabilidade da avaliação vocal. Fatores como experiência do avaliador na área, treinamento auditivo, apoio nas definições transcritas nos protocolos de resposta, orientações e instruções explícitas antes da análise da voz e similaridade entre as respostas dos avaliadores devem ser considerados para que se obtenha um grau de confiabilidade aceitável para a avaliação perceptivo-auditiva da voz ${ }^{5,9,14}$.

Apesar das pesquisas apontarem que a concordância da avaliação perceptivo-auditiva da voz é dependente do tipo de tarefa de fala utilizada vogal sustentada e fala encadeada, optou-se nesta pesquisa por trabalhar com ambas as tarefas na avaliação, uma vez que esse tipo de amostra representa a rotina clínica fonoaudiológica ${ }^{5,15}$.

Estudos revelam que a análise perceptivo-auditiva, de uma forma geral, apresenta boa confiabilidade para diversos métodos utilizados, em especial para avaliações que utilizam a escala GRBAS $^{7-9}$. 
Entretanto, outros autores revelam que mesmo após treinamento auditivo, a concordância interavaliadores para a avaliação vocal é reduzida ${ }^{16}$.

Dados objetivos obtidos por meio da análise acústica complementam a avaliação perceptivoauditiva da voz. A análise espectrográfica é uma das principais ferramentas desta forma de análise e, apesar de ser considerada objetiva, nela se realiza uma avaliação perceptivo-visual do traçado espectrográfico, por meio de uma representação gráfica que traduz os padrões sonoros em padrões visuais $^{10-13}$.

A fim de aprimorar a avaliação fonoaudiológica, estudos descrevem a correlação entre as análises vocais perceptivo-auditivas e perceptivo-visuais do traçado espectrográfico. Apesar de não haver correlação direta entre dados acústicos e auditivos, a presença de determinadas características vocais são observadas na representação espectrográfica, de forma que o uso complementar de ambas as formas de análise proporciona um conhecimento mais preciso da dinâmica vocal e das condições da laringe $\mathrm{e}^{1,7,11-13}$.

Em relação às variáveis que compõem a escala GRBASI, a literatura descreve sobre a facilidade ou dificuldade em analisar e graduar alguns dos seus parâmetros perceptivo-auditivos, justificando a maior ou menor concordância obtida inter e intra-avaliadores.

Segundo estudos, a variável G (grau geral da disfonia) é a mais confiável da escala, pois oferece uma percepção global da disfonia e por isso apresenta maior confiabilidade ${ }^{7,8}$. Outros estudos relatam que os parâmetros perceptivo-auditivos $G$ (grau geral da disfonia), A (astenia) e R (rugosidade) são frequentemente usados e recomendados como uma dimensão básica para caracterizar vozes disfônicas, nesses casos apresentando maior concordância $^{10}$. Não foram encontradas pesquisas que justificassem a maior ou menor concordância obtida para a avaliação das variáveis $\mathrm{B}, \mathrm{S}$ e I.

O desenvolvimento desta pesquisa foi motivado pelo estudo de Martens et al $(2007)^{10}$ que verificaram a interferência do apoio visual do traçado espectrográfico na concordância inter-avaliador na análise perceptivo-auditiva da voz na análise de 70 vozes disfônicas, vogal sustentada e fala encadeada, por seis profissionais com experiência na área, com a escala GRBASI.

O presente estudo manteve o mesmo desenho metodológico do trabalho acima citado, porém, analisou também a concordância intra-avaliador, além de trabalhar com um maior número de vozes, definidos a partir de cálculo amostral com um poder estatístico de $80 \%$, a fim de se verificar a real inter- ferência do apoio visual do traçado espectrográfico na análise perceptivo-auditiva da voz.

Os resultados encontrados nessa pesquisa indicam que não houve diferença estatisticamente significante entre as avaliações perceptivo-auditivas inter-sujeitos com e sem a leitura espectrográfica. Desta forma, pode-se dizer que o apoio visual não aumenta a confiabilidade da avaliação perceptivoauditiva da qualidade vocal, entretanto, aumenta a concordância entre avaliadores para as variáveis G, R, B e S (tabela 1 e figura 2). O apoio visual do espectrograma, portanto, auxilia a avaliação perceptivo-auditiva da qualidade vocal, sem, entretanto, aumentar sua confiabilidade.

As variáveis $A$ (astenia) e $S$ (tensão) são representadas no espectrograma pelo grau de escurecimento do traçado, mas são características preponderantemente perceptivo-auditivas, sendo de difícil percepção na análise espectrográfica ${ }^{12,13}$. Ainda assim, no presente estudo verificou-se um aumento da concordância inter-avaliadores para a variável S quando a análise perceptivo-auditiva foi realizada com o apoio visual do espectrograma. O parâmetro I (instabilidade), por sua vez, é facilmente percebido no espectrograma, de forma que qualquer irregularidade no traçado espectrográfico pode ser interpretada como instabilidade, mesmo que tal característica não seja facilmente percebida auditivamente. Tal fato pode justificar a redução da concordância para esta variável.

Tais achados são discordantes da literatura, na qual se observa melhora significante da concordância inter-avaliador da análise perceptivo-auditiva quando realizada com o apoio visual do espectrograma para os parâmetros perceptivo-auditivos $G, R$ e $B$, em relação aos resultados da análise perceptivo-auditiva sem o apoio visual ${ }^{10}$.

As diferenças de resultados encontradas em ambas as pesquisas podem ser justificadas pelo fato do atual estudo ter um maior poder estatístico com relação ao tamanho da amostra do que o trabalho de Martens et al (2007) ${ }^{10}$.

Deve-se também levar-se em consideração a interferência da experiência dos avaliadores nos resultados encontrados, já que a literatura aponta que o tempo de experiência dos avaliadores impacta nos resultados da análise perceptivo-auditiva ${ }^{5,9,14}$. O presente estudo trabalhou com seis fonoaudiólogos com um a 15 anos de experiência enquanto Martens et al $(2007)^{10}$ trabalharam com o mesmo número de avaliadores com tempo de experiência superior (nove a 20 anos). Pesquisas futuras com um maior controle de tal variável são importantes para a melhor compreensão de tais resultados.

A tabela 2 e o gráfico 2 demonstram que não houve diferença estatisticamente significante entre 
as avaliações perceptivo-auditivas intra-sujeitos sem e com o apoio visual do espectrograma, entretanto, houve aumento da concordância intra-avaliadores após a apresentação do estímulo visual para as variáveis $\mathrm{G}, \mathrm{B}$ e I. Da mesma forma como observado para os valores inter-avaliadores, o apoio visual do espectrograma auxilia a avaliação perceptivo-auditiva da qualidade vocal, sem, entretanto, aumentar sua confiabilidade de forma significante.

Não foram encontrados na literatura trabalhos com a mesma metodologia deste estudo para a comparação de todos os resultados. Porém, pode-se explicar os achados de redução da concordância para o parâmetro $A$ ie ausência de alteração da concordância para $S$ durante a avaliação com apoio visual do espectrograma, pela dificuldade em identificar tais parâmetros perceptivo-auditivos no traçado espectrográfico ${ }^{12,13}$. A redução na concordância do parâmetro $R$ com o apoio visual intra pode ser devido ao aumento de informações oferecidas pelo espectrograma, que pode ter gerado dúvida durante a análise da qualidade vocal, como verificado em outro estudo ${ }^{17}$.

A condição multidimensional da voz e a percepção da qualidade vocal pelos ouvintes, envolvem uma combinação específica de um padrão de processamento holístico associado à análise das características do estímulo ${ }^{5}$.

Todos estes aspectos tornam o estudo da análise perceptivo-auditiva um grande desafio e pesquisas futuras que visem verificar a confiabilidade de tal análise são importantes para o desenvolvimento da avaliação fonoaudiológica.

\section{CONCLUSÕES}

O apoio visual do espectrograma melhorou a concordância inter-avaliadores para a análise perceptivo-auditiva dos parâmetros G (grau geral da disfonia), R (rugosidade), B (soprosidade) e S (tensão), entretanto o aumento da concordância não foi estatisticamente significante.

O apoio visual do espectrograma melhorou a concordância intra-avaliadores para a análise perceptivo-auditiva dos parâmetros G, B e I, entretanto o aumento da concordância não foi estatisticamente significante.

O apoio visual do espectrograma não aumenta significativamente a confiabilidade da avaliação perceptivo-auditiva da voz, mas a auxilia, uma vez que promove um aumento das concordâncias inter e intra-avaliadores.

\begin{abstract}
Purpose: to evaluate the concordance of the auditory-perceptive evaluation between intra and interraters, done as an isolated form and simultaneously accomplished to the correspondent spectrographic tracing in order to check whether the presentation of the vocal stimulus and spectrographic together increases the perceptual voice evaluation reliability. Method: this is a longitudinal study, where six speech and language therapists evaluated in two distinct moments 105 dysphonic and non-dysphonic voices of a perceptual voice evaluation form: firstly without, and afterwards with correspondent spectrograms presentations. Twenty percent of the voices were repeated randomly in both instances, in order to check for the intrarater agreement. GRBASI scale was used for the vocal evaluation. For inter-rater reliability analysis was used the statistical index Kappa Fleiss, and for intrarater reliability was used the Spearman's coefficient correlation. Results: in the perceptual voice evaluation there was not statistically significant difference among inter-rater reliability with and without the spectrographic. However, there was an increase of the concordance among appraisers for $G, R, B$ and $S$. There was no statistically significant difference among intrarater reliability with and without the visual support of spectrogram, however, there was an increase in the intrarater concordance for the variables $G$, $B$ and I, after presenting the visual stimulation. Conclusion: spectrogram's visual support does not significantly increase the perceptual voice evaluation reliability, but helps it, once that it promotes better inter and intra-appraisers' concordance.
\end{abstract}

KEYWORDS: Voice; Voice Disorders; Speech Language and Hearing Sciences; Speech Acoustics 


\section{REFERÊNCIAS}

1. Behlau M, organizadora. Voz: o livro do especialista. Rio de Janeiro: Revinter; 2001.

2. Colton $\mathrm{RH}$, Casper KJ. Compreendendo os problemas de voz. Porto Alegre: Artes Médicas, 1996, 260p.

3. Kreiman J, Gerrat B, Ito M. When and why listeners disagree in voice quality assessment tasks. J Acoustic Soc Amer 2007; 122(4):2354-64.

4. Eadie TL, Baylor CR. The effect of perceptual training on inexperienced listeners' judgments of dysphonic voice. J Voice. 2006;20(4):527-44.

5. Patel S, Shrivastav R. Perception of dysphonic vocal quality: some thoughts and research updates - perspectives on voice and voice disorders. ASHA Division 3. 2007; 17(2): 3-6.

6. Hirano M. Clinical examination of voice. New York: Springer Verlag, 1981, 81-4.

7. Dejonckere PH, Leback J. Acoustic, perceptual, aerodynamic and anatomical correlations in voice pathology. ORL J Otorhinolaryngol Relat Spec 1996;58:326-32.

8. Ma, E. P.-M., \& Yiu, E. M.-L. Multi-parametric evaluation of dysphonic severity. Journal of Voice; 2006;20(3), 380-90.

9. Sellars C, Stanton AE, Mcconnachie A, Dunnet $\mathrm{CP}$, Chapman LM, Bucknall CE, Mackenzie K. Reliability of perceptions of voice quality: evidence from a problem asthma clinic population. The Journal of Laryngology \& Otology 2009; 123, 755-63.
10. Martens JF, Versnel H, Dejonckere PH. The Effect of Visible Speech in the Perceptual Rating of Pathological Voices, Arch Otolaryngol Head neck surg/vol 2007; 2, 133-4.

11. Pontes PAL, Vieira VP, Gonçalves MIR, Pontes AAL. Características das vozes roucas, ásperas e normais: análise acústica espectrográfica comparativa. Rev Bras Otorrinolaringol 2002;68(2):182-8.

12. Batalla FN, Santos PC, Santiago GS, González $B S$, Suàrez Nieto C. Evaluación perceptual de La disfonía: correlación con los parâmetros acústicos y fiabilidad. Acta Otorrinaringol Esp 2004;55:282-7. 13. Drumond LB, Gama ACC. Correlação entre dados espectrográficos e perceptivo-auditivos de vozes disfônicas. Fono Atual 2006; 8(35):49-58.

14. Kelchner LN, Brehm SB, Weinrich B, Middendorf J, Alarcon A, Levin L, Elluru R. Perceptual Evaluation of Severe Pediatric Voice Disorders: Rater Reliability Using the Consensus Auditory Perceptual Evaluation of Voice. J Voice, jan 2009, in press.

15. Bele IV. Reliability in perceptual analysis of voice quality. J Voice. 2005;19(4):555-73.

16. Dornelles S, Jotz GP, Guilherme A. Capacidade discriminatória e confiabilidade da análise perceptiva auditiva da voz de crianças institucionalizadas. In: Ferreira LP, Costa HO. Voz ativa falando sobre a clínica fonoaudiológica. São Paulo: Rocca; 2001. p. 167-81.

17. Valentim A, Côrtes M, Gama ACC. Análise acústica espectrográfica da voz: efeito do treinamento visual na confiabilidade dos resultados. Rev Soc Bras Fonoaudiologia, 2010, no prelo

DOI: 10.1590/S1516-18462010005000123

RECEBIDO EM: 23/04/2010

ACEITO EM: 20/07/2010

Endereço para correspondência:

Ana Cristina Côrtes Gama

Av. Alfredo Balena, 190 - Sala 69

Belo Horizonte - MG

CEP: 30130-100

E-mail: anacgama@medicina.ufmg.br 